\title{
The use of thermally expandable microcapsules for increasing the toughness and heal structural adhesives
}

\author{
Guillaume Pesquet \\ ENSIETA, 2 rue François Verny, 29806 Brest, France
}

Lucas F. M. da Silva

Departamento de Engenharia Mecânica, Faculdade de Engenharia da Universidade do Porto, Rua Dr. Roberto Frias, $4200-465$ Porto,Portugal; lucas@fe.up.pt

Chiaki Sato

Precision and Intelligence Laboratory, Tokyo Institute of Technology, 4259 Nagatsuta, Midori-ku, Yokohama 226-8503, Japan

\begin{abstract}
In this research, the effect of thermally expandable microcapsules (TEMs) on mode I fracture toughness of structural adhesives were investigated. The single-edge-notch bending (SENB) test was used. Firstly, a standard toughness test was performed on adhesives with microcapsules. Secondly, since TEMs start their expansion at approximately $60^{\circ} \mathrm{C}$, the next specimens were fatigue tested expecting a local heating in the notch leading to the desired expansion before being statically loaded for fracture toughness determination. Thirdly, a manual local heating at $90^{\circ} \mathrm{C}$ was applied in the notch before the fracture static test. The experimental results were successfully cross-checked through a numerical analysis using the virtual crack closure technique (VCCT) based on linear elastic fracture mechanics (LEFM). The major conclusion is that fracture toughness of the modified adhesives increased as the mass fraction of the TEMs increased.
\end{abstract}

KEYwORDs. Epoxy/epoxides; Fracture mechanics; Finite element stress analysis; Thermal analysis; Thermally expandable microcapsules (TEMs).

\section{INTRODUCTION}

$\checkmark$ hermally expandable microcapsules, thermally expansive particles, thermo-expandable microsphere (TEMs), or a combination of these words, are all particles made up of a thermoplastic shell filled with liquid hydrocarbon. On the action of heat, the shell softens and the liquid hydrocarbon boils. The hydrocarbon gas works as a blowing agent because the shell expands as the inner pressure increases. The growth in volume can be from 50 to 100 times. This capability brings new and quite unique possibilities for engineers. Because of its high sensitivity to temperature, storage at over $40^{\circ} \mathrm{C}$ is not advised [1].

The use of TEMs in adhesives has allowed industry to have easily dismantlable joints. The simple heating of the joint over $100^{\circ} \mathrm{C}$ leads to an easy separation of the bonded materials. The adhesive expansion may be up to $400 \%$ according to the study of Nishiyama and Sato [2]. This technique is promising because it does not need much time to make this kind of joint and greatly facilitates the dismantling. TEMs are mixed with the resin before the manufacture of the joint. Since they 
expand throughout the joint on the action of heat, they may dismantle the joint. A low fraction of TEMs does not allow the dismantling of the joint and a too high fraction reduces the bond strength. Thus, 5 to $15 \%$ [3] of TEMs in terms of weight fraction is generally considered for this use. It was even shown that the shear strength of a common lap joint increased thanks to microcapsules [1]. Obviously, resin and particles must match to permit the dismantling. Theoretically, matching means here that the resin must be in its rubbery plateau when particles expand to allow its expansion. The microcapsules cannot transmit more than 1 or $2 \mathrm{MPa}$ to the resin [2]. This means that the temperature of expansion of the particles must be slightly higher than the glass transition temperature $\left(T_{g}\right)$ of the resin. Thus, the joint can dismantle by itself or with a low force. If it is not the case, i.e. the $T_{g}$ of the resin is higher than the temperature of expansion of the TEMs, the joint cannot be dismantled. However, one can distinguish at least two cases. In the first, the $T_{g}$ of the resin is much higher than the temperature of expansion of the TEMs and the strength of the adhesive does not permit the expansion of the particles. In the second case, the $T_{g}$ of the resin is slightly higher than the temperature of expansion of the TEMs. In this last case, the stress increase due to the expansion of the microcapsules throughout the resin might create a kind of hydrostatic pressure and will affect its properties and its mechanical behaviour. An increase of hydrostatic stress leads to an increase of elastic limit and failure load [4]. The expansion of the particle would also possibly cause a crack barrier which would increase the fracture toughness of the adhesive. The propagation of cracks brings energy dissipation, hence local heating, especially in dynamic conditions such as in a fatigue test. This local heating may lead to the expansion of the TEMs and thus to a local healing by creation of hydrostatic pressure of crack barrier. In other words, a self-healing or self-toughening of the modified adhesive would occur. The obvious capacity of living beings to heal themselves was transposed recently to polymers [5] and polymer composites [6, 7]. It means that a secondary function of healing is embedded into the adhesive and the reaction of healing is triggered by the damage of the material. So it permits counteracting the service degradation whilst still achieving the primary structural requirement. The healing must lead to a local restoration of mechanical properties. The concept of a self-healing material already exists using numerous ways such as bleeding capsules [5] or hollow fibres [8]. It relies on the microencapsulation of a monomer spreading into cracks when ruptured. The polymerisation starts with the contact of the monomer with a dispersed particulate catalyst dispersed in the resin. Thus, repair self occurs when a crack initiates. This concept of self-healing might also occur in the case of TEMs. Note that if heat must be provided to enhance the mechanical properties of the composite TEMs-resin, then an artificial healing is taking place and not a self-healing. These ideas need to be confirmed or invalidated by experiments, which was the aim of the present investigation.

Assuming that TEMs may modify the fracture toughness by increasing the resistance to crack propagation, one has to choose a proper test to quantify this change. Microcapsules expansion is wanted, and it would be even better if the test itself could lead to the expansion of the microcapsules. An idea is to use mechanical work in the material in order that it generates heat by itself, as fatigue does. Therefore, self-heating by fatigue was considered. Nevertheless, this local generated heat has to be well contained and not transfer to the full specimen. An adhesive joint between metallic adherends such as the double cantilever beam (DCB) test defined in standard ASTM D3433 was not considered because of the high conductivity of metals. Composite adherends, which are less conductive, could have been used but to avoid interaction of adherends in a first analysis, a single-edge-notch bending (SENB) test (ASTM D5045) using a bulk specimen was selected. The test might not bring enough heat to expand the particles. Therefore, a solution to heat the joint must be defined and validated. Moreover, the concentration of TEMs in the resin affects directly the results. Several concentrations and different heating methods were tested. To exhibit differences brought by microcapsules, three types of tests were carried out.

\section{EXPERIMENTAL DETAILS}

\section{Materials}

he diameter of these microcapsules ranges mainly from 10 to $20 \mu \mathrm{m}$ with an average of $15 \mu \mathrm{m}$ and the shell thickness from 3 to $4 \mu \mathrm{m}$ at room temperature. Data from the manufacturer (Matsumoto Yushi-Seiyaku Co., Japan) is given in Tab. 1. These particles start to expand at $60^{\circ} \mathrm{C}$.

Epoxy resins are the most common structural adhesives. The following points were taken into account in the selection of the epoxy resins. The cure temperature must be lower than the temperature expansion of particles. The $T_{g}$ of the adhesive should be around the temperature of expansion of particles to maximise the effect of TEMs on adhesive. In light of these facts, the adhesives presented in Tab. 2 were selected. The tensile stress-strain curves for the three used adhesives are presented Fig. 1. These curves were determined on bulk dogbone samples. These curves show that adhesive AV138M is 
brittle, adhesive 2015 is ductile and adhesive AW106 is very ductile. The several mass fractions that were used are summarized in Tab. 3.Up to $25 \%$ of TEMs were added to the adhesives.

\begin{tabular}{cc}
\hline Particle size & $10-20 \mu \mathrm{m}$ \\
Shell thickness & $3-4 \mu \mathrm{m}$ \\
Density & $1.13 \mathrm{~g} / \mathrm{cm}^{3}$ \\
Chemical composition of shell & Vinylidene-chloride-copolymer \\
Chemical composition of core & Isobutane \\
\hline
\end{tabular}

Table 1: Characteristic of the TEMs (F-30D) used in the present study.

\begin{tabular}{cc}
\hline Adhesive w/ hardener & Glass transition temperature $\left({ }^{\circ} \mathrm{C}\right)$ \\
Araldite AV138M/HV998 & 80 \\
Araldite 2015 & 67 \\
Araldite AW106/HV953U & 45 \\
\hline
\end{tabular}

Table 2: Adhesives selected with indication of $T_{g}$.

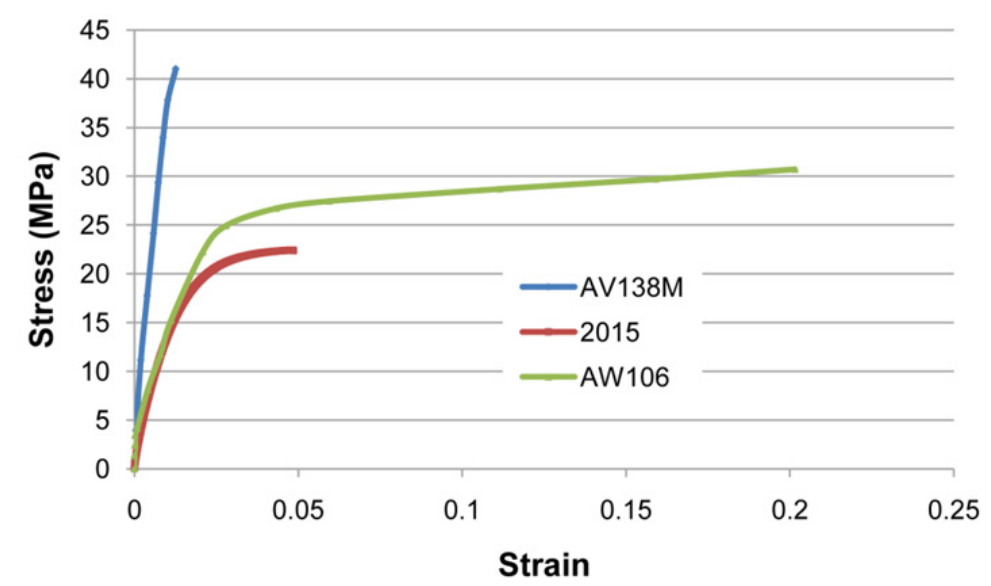

Figure 1: Tensile stress-strain curves of the epoxy adhesives without particles.

\begin{tabular}{cccc}
\hline AV138M/HV998 & $0 \mathrm{wt}^{\%} \%$ & $10 \mathrm{wt}^{\mathrm{0}} \%$ & $20 \mathrm{wt} \%$ \\
Araldite 2015 & $0 \mathrm{wt} \%$ & $10 \mathrm{wt} \%$ & $20 \mathrm{wt} \%$ \\
AW106/HV953U & $0 \mathrm{wt} \%$ & $25 \mathrm{wt} \%$ \\
\hline
\end{tabular}

Table 3: Mass fractions studied for the three adhesives.

\section{Specimens manufacture}

A critical point in this study is the mixing of TEMs in the adhesive. For that purpose, a mixer relying on centrifugal force was used (DAC 150.1 FVZ Speedmixer, Hauschild, Germany). This allows an efficient and homogeneous dispersion, creating visibly bubble-free mixing. The mixture was checked through a scanning electron microscope (SEM) analysis. Small samples were immersed in liquid nitrogen before being fractured. At this temperature, the materials were very brittle and the fracture surfaces were very neat. The particles were always well dispersed. The mixture was poured in a mould consisting in a silicone rubber and a metallic frame [9]. A pressure of $2 \mathrm{MPa}$ was applied in a hot press to the mould. All specimens were cured at $40^{\circ} \mathrm{C}$ at varying times (16h for AV138M, $4 \mathrm{~h}$ for 2015 and $6 \mathrm{~h}$ for AW106) to ensure complete cure. The specimens were machined afterwards from the bulk plates. 


\section{Expansion test}

The expansion with temperature of the neat and modified adhesives (Tab. 3) was measured to check the behaviour with temperature of the adhesives. Small samples $\left(15 \times 10 \times 10 \mathrm{~mm}^{3}\right)$ were heated from room temperature to $105^{\circ} \mathrm{C}$ by step of $5^{\circ} \mathrm{C}$ during 30 mins using an oven. After each temperature step, the specimens were cooled by natural convection. The upper surfaces of the dices were captured with a charge coupled device (CCD) camera through a stereo microscope. Pictures were analysed and the area of the dice calculated for each temperature. Then, 2D expansion of the upper surface was calculated.

\section{Bending test}

Bulk single-edge-notch bending (SENB) specimens (Fig. 2) in accordance with ASTM D5045 were tested in three point bending. All specimens were in accordance with Eq. (1) to have comparable results.

$$
0.45<a / W<0.55
$$

Three specimens were tested for each case (Tab. 3) corresponding to the following conditions:

1. The first specimen was manually saw up to meet the desired testing range relation for $a$. The thickness of the blade was $0.5 \mathrm{~mm}$. In the bottom of the notch, a plastic impression was made by manually pushing in a fresh razor blade. Then, specimens were tested. A natural crack is recommended by standard ASTM D5045 but the creation of a natural crack is very difficult for brittle adhesives such as adhesive AV138M.

2. The second specimen was pre-fatigued to open a natural crack before testing it statically to fracture. The fatigue crack was propagated under displacement control conditions up to meet the desired testing range relation with a high frequency to self heat the sample $(20 \mathrm{~Hz})$. The specimens were painted in matt black and an increase in macroscopic temperature was monitored with a thermographic camera (Fluke Ti25) with an accuracy of $\pm 2{ }^{\circ} \mathrm{C}$ and a thermal sensitivity lower than $0.1^{\circ} \mathrm{C}$.

3. The third specimen was saw and received a plastic impression as the first specimen. Next, a blade at $90^{\circ} \mathrm{C}$ was inserted into the notch for 5 minutes, applying a controlled local heating with a copper sheet of $0.1 \mathrm{~mm}$ thickness that was carefully applied in the bottom of the notch. The temperature was controlled using a thermocouple and a thermographic camera. Fig. 3 shows the local heating using thermal imaging. One hour later, when fully cooled by natural convection, the specimen was tested statically to failure. Results are wholly comparable with the first specimen, the only difference being the heating step.

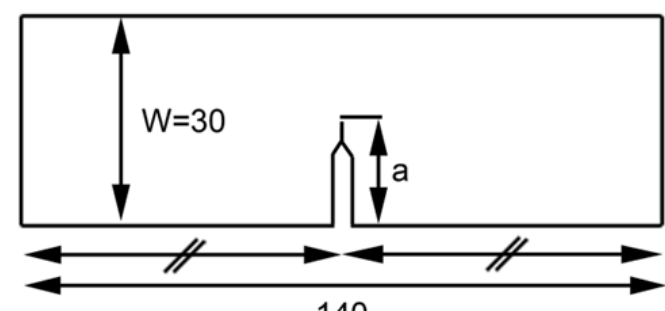

140



Figure 2: Single edge notch three point bending test specimen (dimensions in $\mathrm{mm}$ ).

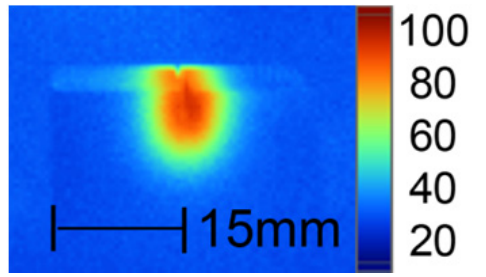

Figure 3: Thermal image of a heated notch with a copper sheet at $90^{\circ} \mathrm{C}\left(\right.$ temperature scale in $\left.{ }^{\circ} \mathrm{C}\right)$.

The tests were carried out in a MTS servo-hydraulic machine with a load-cell of $5 \mathrm{kN}$ load in laboratory ambient conditions $\left(25^{\circ} \mathrm{C}\right.$ and relative humidity of $\left.50 \%\right)$ and a cross-head rate of $10 \mathrm{~mm} / \mathrm{min}$.

The crack length was measured with a stereo microscope having a micrometer screw, being able to measure the crack on the two sides of the specimen. After failure, a CCD camera through a stereo microscope was used to observe all fracture surfaces. 
Typical load-displacement curves are shown in Fig. 4 for adhesive 2015. The load-displacement curves were almost linear up to fracture despite the non-linear behaviour exhibited in the tension specimens. Even very ductile adhesives provide linear curves through the SENB test. Similar bending tests were conducted successfully by Chen et al. [10] with a carboxyl-terminated butadiene nitrile rubber $(\mathrm{CTBN})$ adhesive that exhibits a large strain to failure $\left(\varepsilon_{\max }=14 \%\right)$. The fracture toughness was calculated using the following relationship (ASTM E399-81)

$$
K=\frac{P S}{B W^{3 / 2}} f(a / W)
$$

where $P$ is the failure load, $S$ is the span of the beam specimen, $B$ is the thickness of the specimen, $W$ is the width of the specimen, $a$ is the crack length and the function $f$ is as $f(x)=2.9 x^{1 / 2}-4.6 x^{3 / 2}+21.8 x^{5 / 2}-37.6^{7 / 2}+38.7 x^{9 / 2}$, where $x$ $=a / W$. All the specimens broke in a brittle manner and the fracture surfaces were flat without shear lips. In order to calculate plane-strain fracture toughness $K_{I c}$, the conditions presented Eq. (3) should be met, where $\sigma_{\max }$ is the tensile strength of the adhesive.

$$
B, a,(W-a)>l=2.5\left(\frac{K}{\sigma_{\max }}\right)^{2}
$$

The criteria described in Eq. (3) were not always met. However, all specimens having the same size, the results remain fully comparable.

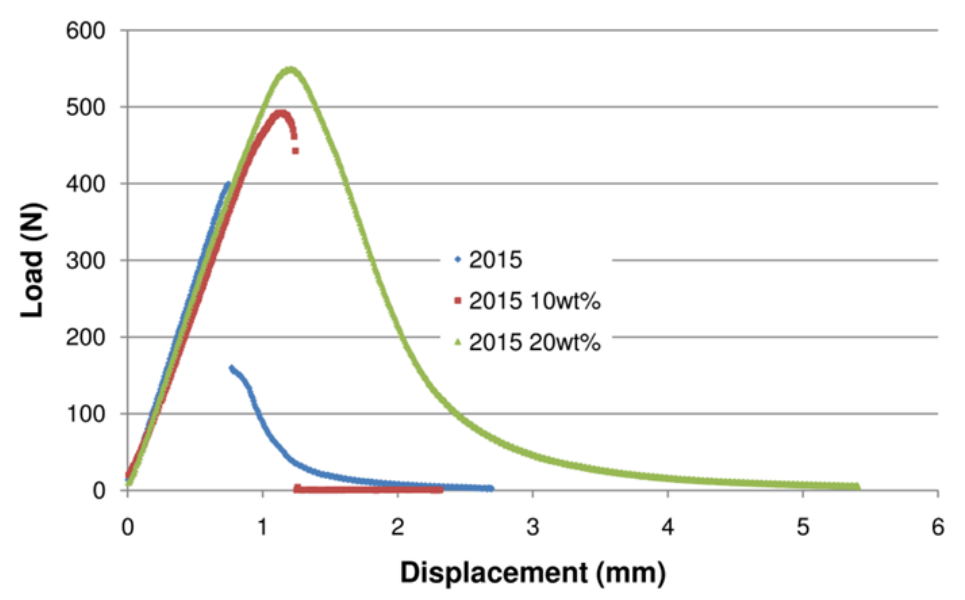

Figure 4: Experimental load-displacement curves for adhesive 2015 (type 1 specimen).

\section{Tensile test}

Tensile tests on dogbone bulk specimens $2 \mathrm{~mm}$ thick and $10 \mathrm{~mm}$ wide were carried to be able to check the plane-strain conditions (Eq. (3)) and assess the effect of the TEMs on the stress-strain characteristics. Tensile tests were only conducted on adhesive 2015 because it might deviate from plane-strain conditions due to its high ductility and low tensile strength. The tests were carried out in a MTS servo-hydraulic machine with a load-cell of $5 \mathrm{kN}$ load in laboratory ambient conditions $\left(25^{\circ} \mathrm{C}\right.$ and relative humidity of $\left.50 \%\right)$ and a cross-head rate of $10 \mathrm{~mm} / \mathrm{min}$.

\section{RESULTS}

\section{Expansion test}

$\mathrm{F}$ ig. 5 shows the expansion of used adhesives with respect to temperature. This expansion was calculated from the area of the upper surface of a dice. This $2 \mathrm{D}$ expansion is defined by the area after the heating step divided by the initial area and is then called relative $2 \mathrm{D}$ size. 


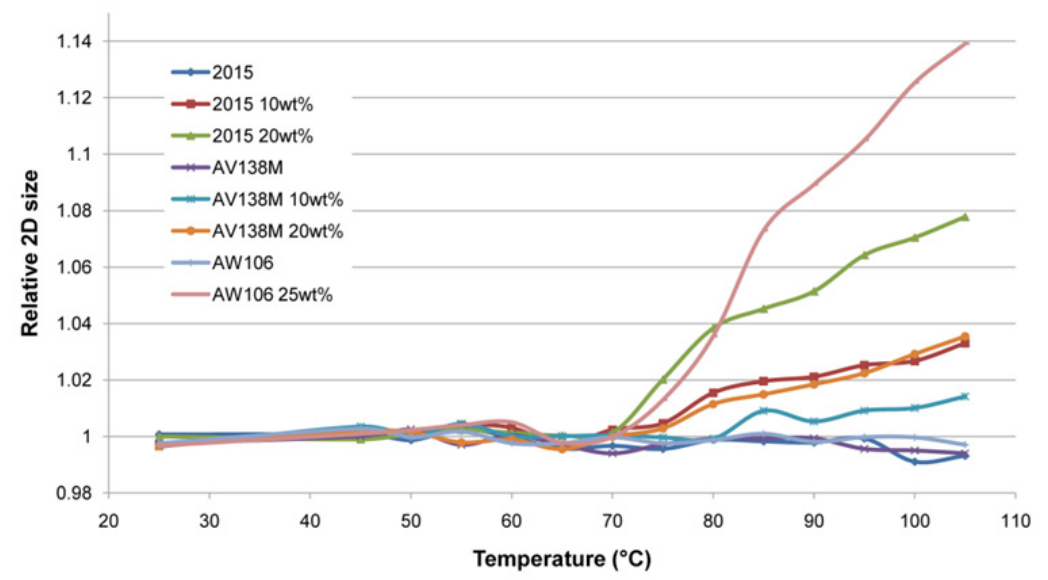

Figure 5: Expansion of neat and modified adhesives as a function of temperature.

The three non-modified adhesives exhibited no expansion. When adhesives without TEMs are heated, they should merely expand according to their volumetric thermal expansion ratio coefficient and then contract to the original shape when naturally cooling. Because all the measurements were done at the same room temperature, all adhesives without TEMs should exhibit no expansion. The three non-modified adhesives even showed a slight decrease in volume. Because they were cured at $40^{\circ} \mathrm{C}$, when they went over $40^{\circ} \mathrm{C}$, they might have cured further. As it is known, adhesive may exhibit some shrinkage after curing. Even if the non-modified adhesives exhibited some slight shrinkage, all the measurements of the three non-modified adhesives remained within a range of $1 \%$. Therefore, the accuracy of the measurements was under a satisfying $1 \%$.

Fig. 5 shows that expansion starts at approximately $70^{\circ} \mathrm{C}$ which is in accordance with the TEMs data sheet and the $T_{g}$ of the adhesives. There is less expansion for adhesive AV138 because its $T_{g}$ is $80^{\circ} \mathrm{C}$ and gives more resistance to expansion than the other adhesives. From $85^{\circ} \mathrm{C}$, all the modified adhesives exhibited more than $1 \%$ of expansion. Therefore, the heating step at $90^{\circ} \mathrm{C}$ for the bending test guarantees that there is expansion of the particles.

\section{Bending test}

A typical fracture surface of specimens sawed and with a natural (pre fatigue tested) is shown in Fig. 6. The fatigue precrack is clearly seen in Fig. 6(b). Also, ductile and brittle fracture areas can be identified.

The values of stress intensity factor $\mathrm{K}$ for each kind of adhesive are plotted as a function of the TEMs mass fraction in Fig. 7, Fig. 8 and Fig. 9. Three curves are plotted for each adhesive corresponding to the three types of specimens (see Section Experimental details: Bending test).


Figure 6: Typical fracture surfaces (adhesive 2015 with $10 \mathrm{wt} \%$ ) for of a specimen with a sawed crack (a) and with a fatigue pre-crack (b).

These figures show that the toughness of the modified adhesives is improved by microcapsules. It is confirmed by every result but the extreme case of AW106 adhesive with $25 \mathrm{wt} \%$. The toughness of specimens having received a plastic impression was always higher than the crack opened with fatigue. That makes totally sense (the required load to break the specimen with a notch is higher than with a natural crack because the stress concentration in a notch is lower than in a 
crack.) if we assume there was no healing effect brought by fatigue. The macroscopic temperature increase obtained by the thermographic camera was always under $2^{\circ} \mathrm{C}$, which is not enough to expand the particles.

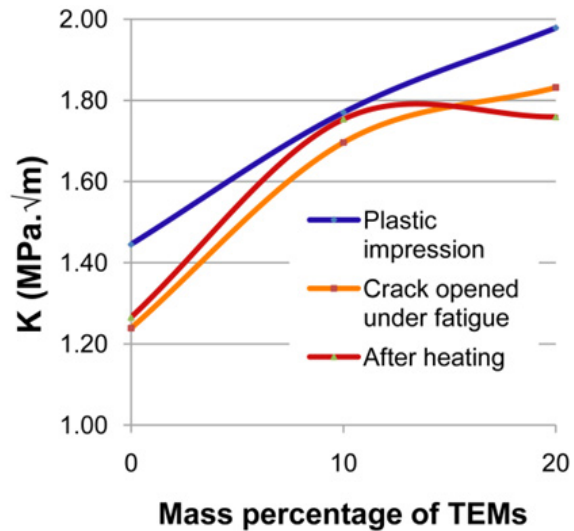

Figure 7: Fracture toughness as a function of TEMs mass fraction for adhesive 2015 .

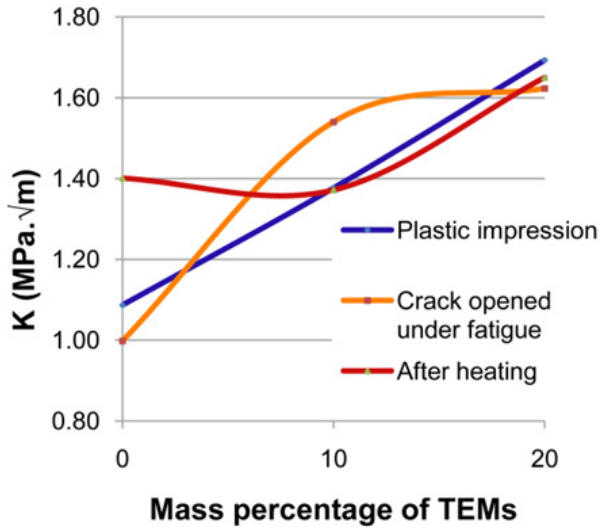

Figure 8: Fracture toughness as a function of TEMs mass fraction for adhesive AV138.

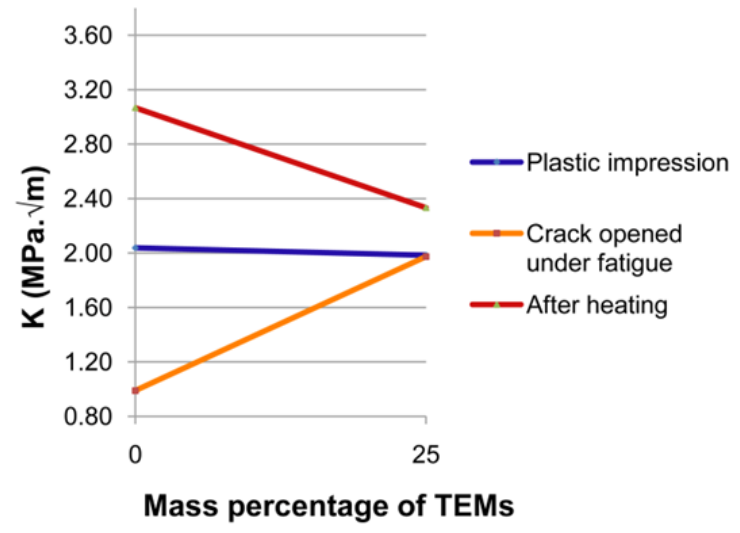

Figure 9: Fracture toughness as a function of TEMs mass fraction for adhesive AW106.

The local heating with the copper sheet expected to heal modified adhesives was unexpectedly highly salutary to nonmodified adhesives AW106 and AV138M. However, the local heating at $90^{\circ} \mathrm{C}$ did not heal the modified adhesives since no increase in toughness was noticed.

Modified adhesives were examined by SEM. Small samples were immersed in liquid nitrogen before being fractured. At this temperature, the materials were very brittle and the fracture surfaces were very neat. Typical fractures surfaces are shown in Fig. 10 for adhesive AW106 with 10\%. It can be seen that the fracture surface is increased by the particles. It is as if the TEMs behave as obstacles for crack propagation. There is a consistent tail phenomenon where all tails go in the same direction.
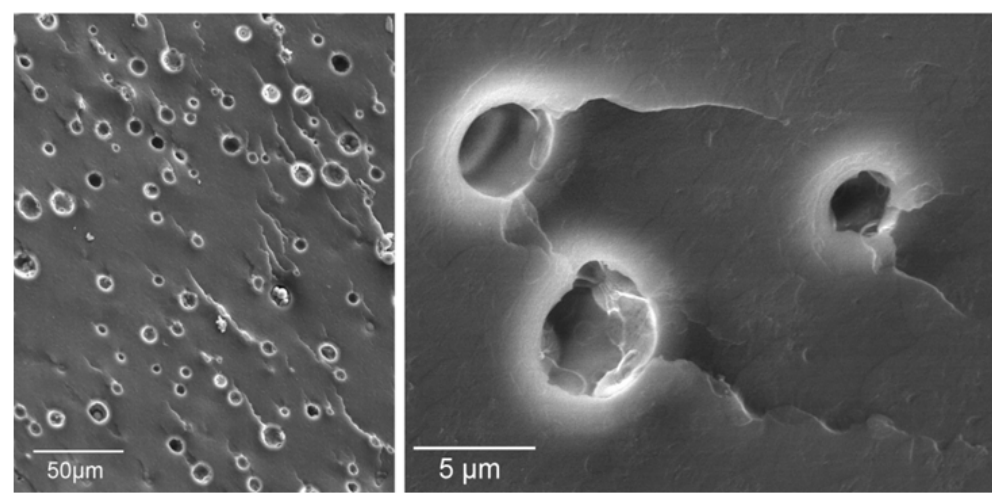

Figure 10: Fracture surface obtained by scanning electron microscopy for adhesive AW106 with 10wt $\%$. 
The TEMs expanded did not increase the fracture toughness of modified adhesives but the simple presence of TEMs did. TEMs are filled with liquid hydrocarbon and are, therefore, probably poorly compressible as rubber particles are. In addition, their size and the mass fractions used are comparable to those of rubber particles. Therefore, the dominant toughening mechanism might be similar to the one that occurs with adhesives modified with rubber particles. The TEMs as a second phase may trigger inelastic processes at a lower macroscopic stress than the stress required to trigger inelastic processes in single-phase adhesives [11]. Therefore, the adhesive is able to absorb the energy of deformation through plastic shear deformation between the particles [12-13].

\section{Tensile test}

Fig. 11 presents stress-strain curves for neat and modified Araldite 2015 adhesive. The TEMs decrease the tensile strength and increase the failure strain. The energy under the tensile curve was computed (toughness modulus) and is presented in Tab. 4 along with other values taken from the tensile curves such as the Young's modulus, the tensile strength and the failure strain. The tensile curves also show that the TEMS increase the toughness and confirm the results obtained with the bending tests.

The response of the modified adhesives shows a drop in stress after the proportional limit after which the stress starts to increase again. The same effect was obtained with rubber particles by Imanaka et al [14].

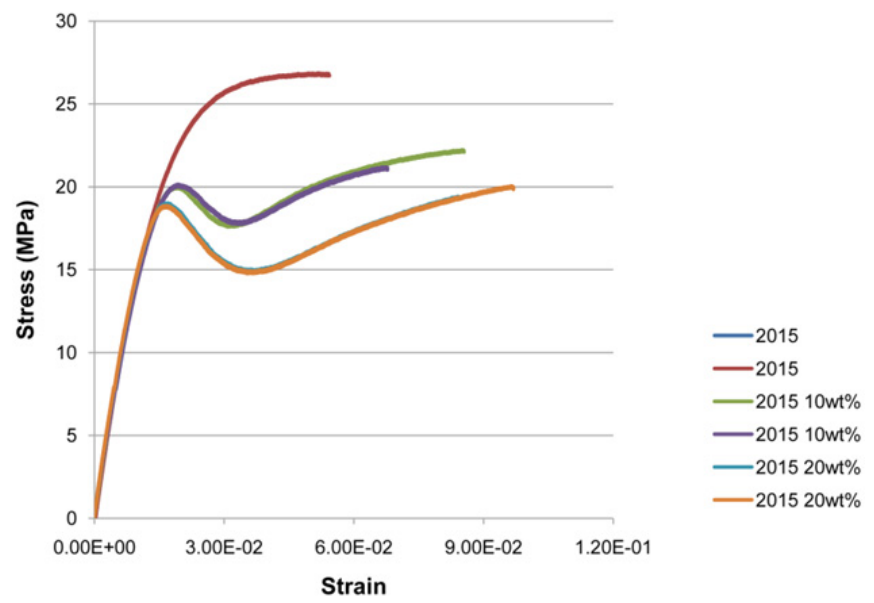

Figure 11: Tensile stress-strain curves for adhesive 2015.

\begin{tabular}{cccc}
\hline Araldite 2015 & $0 \mathrm{wt}^{\%} \%$ & $10 \mathrm{wt} \%$ & $20 \mathrm{wt}^{\%} \%$ \\
$\mathrm{E}(\mathrm{MPa})$ & 1658 & 1565 & 1677 \\
Modulus of toughness $\left(\mathrm{mJ} / \mathrm{mm}^{3}\right)$ & 0.81 & 1.14 & 1.27 \\
$\sigma_{\max }(\mathrm{MPa})$ & 26.9 & 21.8 & 19.8 \\
$\varepsilon_{\max }$ & $5.4 \%$ & $7.7 \%$ & $9.1 \%$ \\
\hline
\end{tabular}

Table 4: Results from the tensile tests on adhesive 2015.

\section{FINITE ELEMENT ANALYSIS}

finite element (FE) analysis of the bending test was carried out. The experimental values of the toughness were the input in the FE analysis and the corresponding numerical load-displacement curves were compared to the experimental ones.

\section{Details of the numerical simulation}

The geometry represented in Fig. 2 was considered. The simulation was conducted assuming linear elastic fracture mechanics (LEFM) and the strain energy release rate was computed using the Virtual crack closure technique (VCCT). The crack was propagated when the strain energy release rate at the crack tip was above the critical strain energy release 
rate of the material in mode I (GIc). VCCT does not need special elements or remeshing but needs two surfaces that are initially partially bonded.

The experimental results showed that the maximum deflection is low compared to the size of the specimen, therefore geometric linearity can be assumed. The maximum deflection (obtained with 2015 20wt\%) of the SENB specimens was always under $3 \mathrm{~mm}$. However, geometric nonlinearity option was used to help the convergence of the VCCT analysis.

Plane strain condition was assumed. Because first-order elements generally work best for crack propagation analysis [15], plain-strain, four-node, linear CPE4 [15] element were selected. 100 elements were used along the height and 100 elements were used along the length of each part with a geometric decrease in size next to the bonded surface. 20000 elements were used in total. The width of elements next to the bonding area was $2.4 \times 10^{-2} \mathrm{~mm}$. VCCT does not exhibit a strong mesh dependency. Two computations were done with the same geometry and material properties but with 360 and 20,000 elements, respectively. The coarse mesh is slightly stiffer but the displacement needed to disbond the first nodes was rather accurately found with the coarse mesh.

\section{Results of the numerical simulation}

Three cases are presented here for illustration purposes: Araldite $20150 \mathrm{wt} \%$, Araldite AW106 25wt $\%$, Araldite AV138M $20 \mathrm{wt} \%$. Similar conclusions were obtained for the other cases. The three experimental load-displacement curves come from SENB specimens with a crack opened under fatigue. For each case, both experimental and numerical curves are plotted for comparison purposes.

The first case presented is for the ductile 2015 adhesive in Fig. 12(a). As VCCT use LEFM approximation, there is no plasticity. That explains that the peak of the experimental curve is smoother than the numerical one. However, the prediction is overall correct. The same conclusion can be drawn from the case of adhesive AW106 with $25 \mathrm{wt} \%$ of TEMs (Fig. 12(b)). The third case is for adhesive AV138M with 20wt\% TEMs in Fig. 12(c). In this case, the experimental failure of the specimen was instantaneous. The starting of disbonding was accurately determined by VCCT. However, there is a softening of the specimen in the numerical simulation that was not captured experimentally.

The numerical results show that modified adhesives can be properly simulated with a homogenous model knowing the critical strain energy release rate. The macroscopic deformation of the experimental specimens was ruled by linear elasticity and the assumption of LEFM was correct for simulating these SENB specimens.
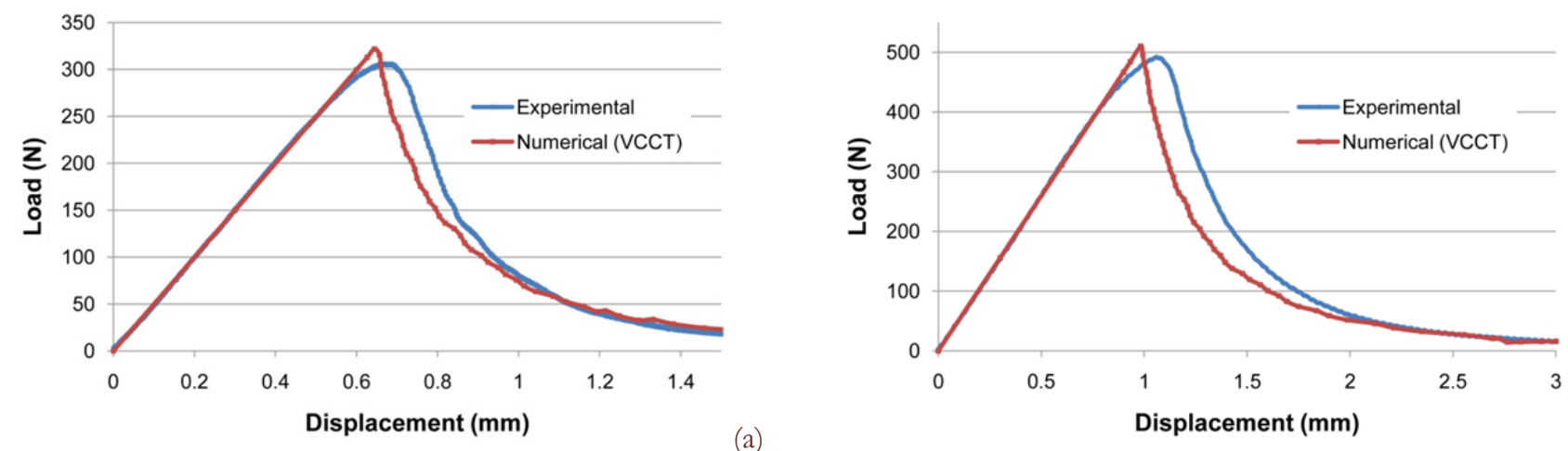

(a)

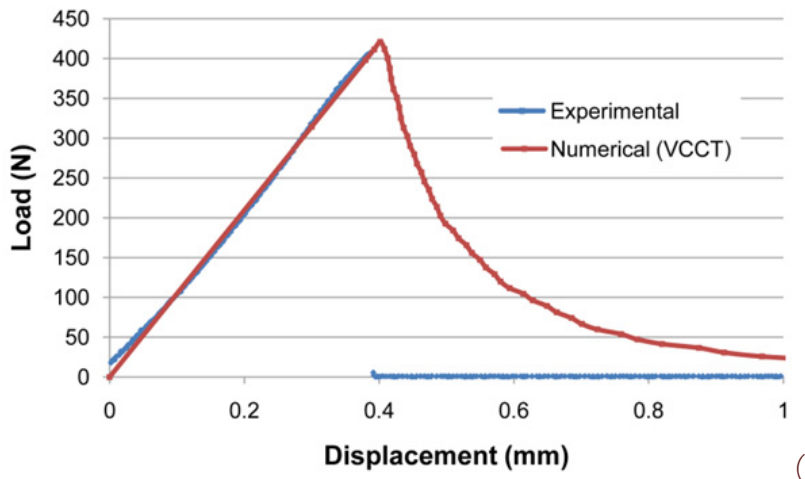

Figure 12: Numerical and experimental load-displacement curves for (a) adhesive 2015 non modified; (b) adhesive AW106 with 25wt $\%$ TEMs; (c) adhesive AV138 with 20wt $\%$ TEMs. 


\section{CONCLUSIONS}

7 he effect of thermally expandable microcapsules (TEMs) on structural adhesives was investigated in terms of mode I fracture toughness. The single-edge-notch-bending (SENB) test was used. Three adhesives were selected (AW106 that is very ductile, 2015 that is ductile and AV138M that is brittle) and different mass fraction of TEMs were studied. Firstly, a standard fracture test was conducted. Secondly, since TEMs expands start their expansion at about $60^{\circ} \mathrm{C}$, specimens fatigue testing was performed expecting a local heating of the notch. Then, the specimen was statically tested. Thirdly, the specimen was heated locally in its notch at $90^{\circ} \mathrm{C}$ to lead to a local expansion of the particles. Then, the specimen was loaded for fracture toughness determination. The following conclusions can be drawn.

1. The fracture toughness increased as the mass fraction of TEMs increased. However, the very ductile adhesive AW106 did not show clearly this increase in terms of toughness.

2. The two local heatings tested did not heal TEMs-modified adhesives.

3. A parallel could be drawn between TEMs and rubber incorporation since the sizes of the particles are comparable and both are barely compressible. Indeed, stress-strain curves for TEMs-modified and rubber-modified exhibit similarities.

4. As rubber particles, TEMs-induced shear yielding of the epoxy matrix is believed to be the dominant toughening mechanism. However, since TEMs are a shell filled with liquid, they may not exhibit the crack-bridging mechanism.

5. The specimens were successfully simulated using linear elastic fracture mechanics (LEFM) approximation. The bending test was simulated with the virtual crack closure technique (VCCT) using the experimental strain energy release rate.

6. TEMs have been developed for recycling purpose by allowing joints to be dismantled. However, TEMs also increase the fracture toughness of adhesives Therefore, TEMs-modified adhesives could be used on a larger scale.

\section{REFERENCES}

[1] Y. Nishiyama, N. Uto, C. Sato, H. Sakurai, Int J Adhesion and Adhesives, 23 (2003) 377.

[2] Y. Nishiyama, C. Sato, In: Adhesion - Current Research and Applications, W. Possart (editor), Wiley, Weinheim, (2005) 555.

[3] H. Ishikawa, K. Seto, S. Shimotuma, N. Kishi, C. Sato, Int J Adhesion and Adhesives, 25 (2005) 193.

[4] J.-Y. Cognard, R. Créachcadec, J. Maurice, P. Davies, M. Peleau, L.F.M. da Silva, J Adhesion Science and Technology, 24 (2010) 1977.

[5] S.R. White, N.R. Sottos, P.H. Geubelle, J.S. Moore, M.R. Kessler, S.R. Sriram, E.N. Brown, S. Viswanathan, Nature, 409 (2001) 794.

[6] R.S. Trask, H.R. Williams, I.P. Bond, Bioinspir. Biomim., 2 (2007) 1.

[7] M.R. Kessler, N.R. Sottos, S.R. White, Composites Part A, 34 (2003) 743.

[8] I.P. Bond, J.W.C. Pang, Compos Part A, 36 (2005) 183.

[9] L.F.M. da Silva, R.D. Adams, J. Adhesion Science and Technology, 19 (2005) 109.

[10] Z. Chen, R.D. Adams, L.F.M. da Silva, Int J Fracture, 167 (2011) 221.

[11]J. Du, M.D. Thouless, A.F. Yee, Int J Fract, 92 (1998) 271.

[12] A.F. Yee, R.A. Pearson, J Mater Sci, 21 (1986) 2462.

[13] Y. Huang, A.J. Kinloch, J.Mater Sci, 27 (1992) 2753.

[14] M. Imanaka, D. Yamashita, Y. Suzuki, A. Fujinami, Polymer \& Polymer Composites, 13 (2005) 765.

[15] ABAQUS Analysis User's Manual. ABAQUS 6.9 (2009). 